\title{
DATACIONES RADIOCARBÓNICAS (AMS) DE RESIDUOS ORGÁNICOS EN TIESTOS DEL BOSQUE NORPATAGÓNICO
}

\author{
RADIOCARBON DATES (AMS) OF ORGANIC RESIDUES \\ IN SHERDS OF THE NORTH PATAGONIAN FOREST
}

\author{
Cristina Bellelli'1 ${ }^{1}$ Pablo M. Fernández² y Mariana Carballido Calatayud ${ }^{3}$ \\ Recibido 30 mayo 2020. Aceptado 18 agosto 2020
}

\begin{abstract}
Resumen: Se presentan los resultados de las dataciones radiocarbónicas obtenidas sobre residuos orgánicos extraídos de cinco tiestos provenientes de tres sitios ubicados en la franja boscosa cordillerana del suroeste de Río Negro y noroeste del Chubut. Son los primeros conocidos para ambientes de bosque y cubren el lapso comprendido entre $960 \pm 22$ y $328 \pm 31$ años AP. Además, estos resultados permitieron discutir cuestiones relacionadas con la integridad y resolución de los depósitos, permitiendo aclarar preguntas acerca de la formación de los sitios planteadas con anterioridad.

Palabras clave: Cronología, cerámica, resolución, poblamiento, bosque norpatagónico.
\end{abstract}

\begin{abstract}
Radiocarbon dates obtained from organic residues deposited on the walls of five sherds recovered at three archaeological sites are presented here. The sites are located in the North Patagonian forest (Chubut and Río Negro Provinces). These dates are the first obtained in a forest environment and cover the period between $960 \pm 22$ and $328 \pm 31$ years BP. Besides, these results allowed us to discuss issues related to the integrity and resolution of archaeological deposits, clarifying previsouly raised questions about site formation processes
\end{abstract}

Keywords: Chronology, pottery, resolution, peopling, North Patagonian forest.

\section{Introducción}

Los residuos derivados de la cocción realizada en ceramios de origen arqueológico nos brindan la oportunidad de acercarnos a su historia de vida, aunque este acercamiento muchas veces está limitado por el estado en que recuperamos la evidencia. En ambientes como el de la franja boscosa lacustre de Norpatagonia, el alto índice de fragmentación y los procesos de meteorización han enmascarado buena parte de esta historia. Sin embargo, en los últimos años los resultados de trabajos que estuvieron dirigidos a conocer las paleodietas y las estrategias de subsistencia a través de los estudios isotópicos y de ácidos grasos han abierto un nuevo camino para superar estas limitaciones (Chaile, Tessone, Cassiodoro, Bellelli \& Belardi, 2018). Este no es el único, ya que aparte de lo que los residuos orgánicos nos permiten conocer sobre la funcionalidad de la evidencia cerámica, son también una vía para establecer cronologías absolutas. Además del cómo y con qué fue usado un ceramio, a través de los fechados realizados sobre este tipo de adherencias es posible, previos los controles tafonómicos necesarios, acercarnos al momento en que estuvo en uso.

En esta oportunidad presentamos cinco fechados directos sobre residuos orgánicos de tiestos recuperados en nuestra área de investigación, comprendida entre el límite sur del Parque Nacional Nahuel Huapi y el límite norte del Parque Nacional Los Alerces y desde la frontera con Chile al oeste hasta el paralelo de $71^{\circ}$ hacia el este (provincias de Río Negro y Chubut) (Figura 1).

Esta información nos permite precisar la resolución y cronología de tres contextos arqueológicos (de norte a sur:
Paredón Lanfré, Campamento Argentino y Cerro Pintado) a partir de una estrategia de fechado que permite datar de manera directa el uso de la tecnología cerámica en estos sitios y en el bosque de esta latitud. A la vez profundiza el conocimiento sobre los aspectos cronológicos del modelo de ocupación del bosque, elaborado a partir de la secuencia de uso humano del área del Manso inferior (Río Negro) y su correlación con la cronología disponible para otros sectores de bosque del centro-norte de la Patagonia argentina (Fernández, Carballido Calatayud, Bellelli \& Podestá, 2013; Fernández et al., 2019a).

1 Instituto Nacional de Antropología y Pensamiento Latinoamericano (INAPL) - Consejo Nacional de Investigaciones Científicas y Técnicas (CONICET). 3 de Febrero 1370 (1426) Buenos Aires crisbellelli@yahoo.com.ar.

2 Instituto Nacional de Antropología y Pensamiento Latinoamericano (INAPL) - Consejo Nacional de Investigaciones Científicas y Técnicas (CONICET) - Universidad de Buenos Aires (UBA). 3 de Febrero 1370 (1426) Buenos Aires - pablomarcelofernand@gmail. com.

3 Instituto Nacional de Antropología y Pensamiento Latinoamericano (INAPL) - Consejo Nacional de Investigaciones Científicas y Técnicas (CONICET) - Universidad de Buenos Aires (UBA). 3 de Febrero 1370 (1426) Buenos Aires - mcarballidocalata@hotmail. com. 


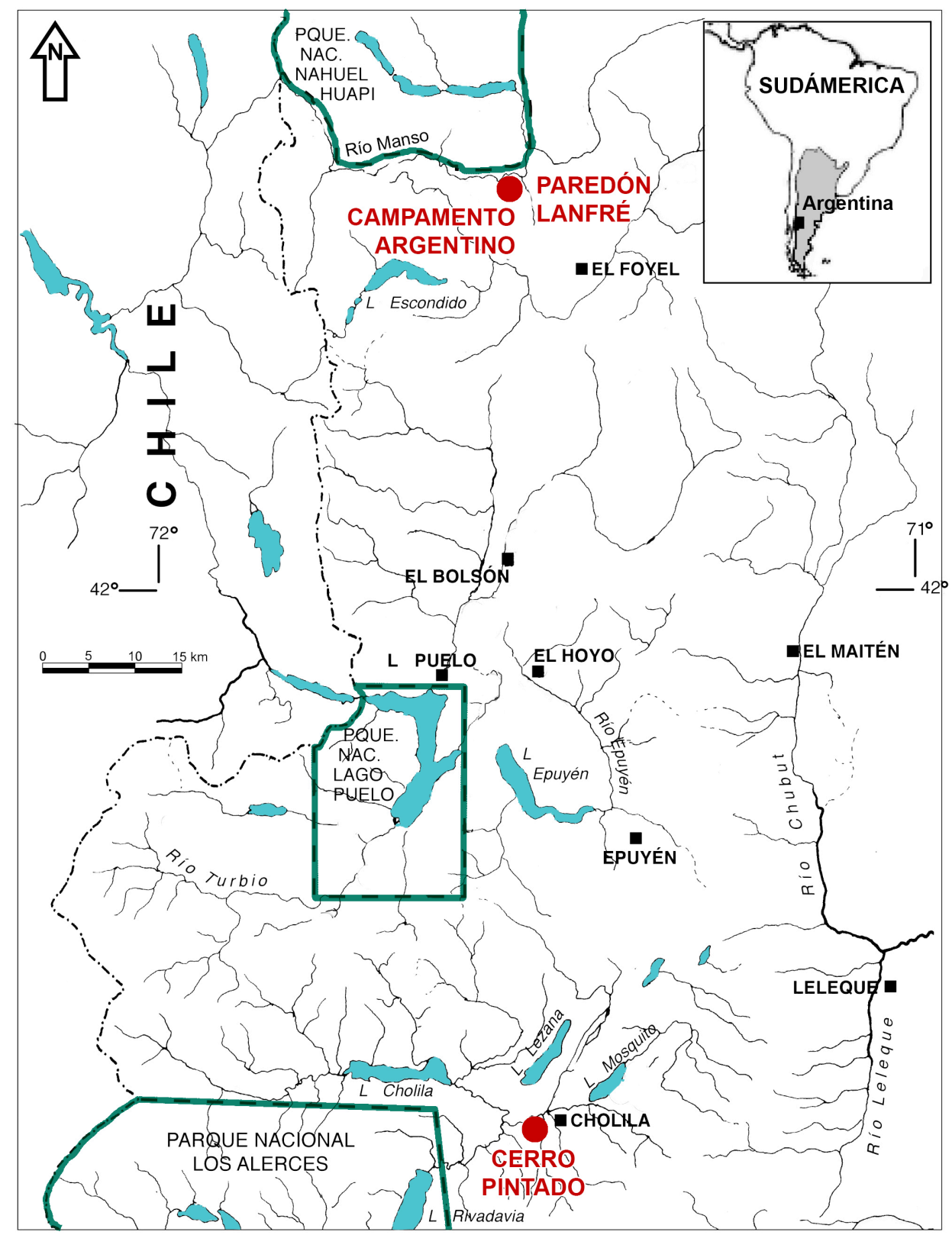

Figura 1. El área de investigación y localización de los sitios de proveniencia de los tiestos analizados.

\section{Antecedentes y presentación del problema}

Las ocupaciones más antiguas registradas en nuestra área de investigación provienen del sitio Población Anticura (valle del río Manso inferior, Río Negro). Allí se registran fechados en el Holoceno temprano $\left(8230 \pm 110\right.$ a $7240 \pm 90$ años ${ }^{14} \mathrm{C}$ AP $)$, el Holoceno medio $\left(4724 \pm 54\right.$ años ${ }^{14} \mathrm{C}$ AP) y el Holoceno tardío inicial $\left(3350 \pm 100\right.$ a $2270 \pm 80$ años ${ }^{14} \mathrm{C}$ AP, Fernández et al., 2013; 2019a), dándole continuidad espacial hacia el sur a los trabajos que años antes habían brindado una profundidad temporal de $10.570 \pm 130$ años ${ }^{14} \mathrm{C}$ AP en el Parque Nacional Nahuel Huapi (Hajduk, Albornoz \& Lezcano, 2006). Hace 2000 años se registra un aumento en la cantidad de sitios y contextos tanto en el Manso inferior como en otros sectores del área de investigación y hacia
1700 años ${ }^{14} \mathrm{C}$ AP se incrementa la recurrencia y/o permanencia de los cazadores en el bosque, modo de ocupación que se prolonga hasta tiempos históricos (Fernández \& Tessone 2014; Fernández et al., 2013, 2019a; Fernández, Carballido Calatayud, Bellelli \& Fernández, 2019b).

Los materiales cerámicos recuperados en el área de investigación provienen de sitios o niveles datados durante el lapso de mayor recurrencia/permanencia dentro del bosque. En el valle del Manso inferior corresponden a los niveles Holoceno tardío final y Tiempos históricos del sitio Población Anticura, a la totalidad de las ocupaciones de los sitios Paredón Lanfré, Campamento Argentino, Casa de Piedra de Carro y a un hallazgo de una vasija casi completa realizado en superficie en cercanías del primer sitio mencionado. En el valle del río Epuyén (Chubut) vienen 
del sitio Risco de Azócar 1 y en Cholila (Chubut) del sitio Cerro Pintado, ambos del Holoceno tardío final (Bellelli, Carballido, Fernández \& Scheinsohn, 2003; Bellelli, Carballido, Fernández \& Scheinsohn, 2007; Podestá et al., 2007; Bellelli 2020a, 2020b).

No obstante, la asignación cronológica de esta tecnología presenta las mismas dificultades que las observadas para el resto de los materiales recuperados en sitios de bosque. Estos contextos están sometidos a complejos procesos de formación que afectan no sólo su integridad sino también su resolución (Carballido Calatayud \& Bellelli 2020; Fernández et al., 2008, 2019b). En general los sitios comprenden depósitos sedimentarios potentes que pueden alcanzar varios metros de profundidad. Estos sedimentos suelen ser homogéneos y, además, los materiales arqueológicos se dan sin solución de continuidad. De allí que la principal estrategia implementada para identificar diferencias temporales ha sido datar materiales a lo largo de todas las secuencias. El fechado de carbón vegetal de estructuras de combustión fue la forma más común de alcanzar este objetivo. Pero no siempre se preservan estas estructuras, de allí que también se han fechado otros materiales y acumulaciones de carbón, aun cuando estas últimas poseen cierto grado de ambigüedad al momento de establecer la relación entre la edad obtenida y las evidencias asociadas (Fernández et al., 2013).

La dinámica de perturbaciones naturales del bosque andino patagónico incluye a los incendios forestales (Whitlock et al., 2006; Giaché \& Bianchi, 2018). Este fenómeno, entre otros efectos, produce una "lluvia natural de carbón", que puede acumularse en rasgos negativos del paisaje o en trampas sedimentarias, como los reparos rocosos en los que se ubican la mayoría de los sitios excavados en el área. Ampliar la estrategia de fechados al incluir los residuos orgánicos de tiestos cerámicos se revela como una forma de superar las limitaciones en las asociaciones cronológicas en estos sitios, al datar de manera directa el uso de esta tecnología y, por ende, la ocupación humana de estos lugares.

\section{Cerámica en el bosque. Características principales}

Las particularidades del ambiente de bosque de Nothofagus sp. - Austrocedrus chilensis que domina el área de investigación, con lluvias que promedian los $2000 \mathrm{~mm}$ anuales y que se hacen más intensas hacia el límite con Chile, y los frecuentes incendios forestales que, de acuerdo con estudios paleoambientales en curso, se dan con diferente intensidad desde que se conformó el bosque actual hace 3500 años (Iglesias, Whitlock, Bianchi, Villarosa \& Outes, 2012; Giaché \& Bianchi, 2018), inciden fuertemente en la formación de los depósitos, en su resolución e integridad y en la preservación de la evidencia (Carballido Calatayud \& Bellelli, 2020). Así, la muestra cerámica de los sitios del valle de los ríos Manso y Epuyén, se ve alterada principalmente por la humedad y por el fuego. El redondeamiento y pulido de buena parte de los bordes fracturados y la pérdida parcial de las superficies apuntan a la acción del agua, tanto in situ como a través del transporte, mientras que cambios de color, saltaduras y/o adherencias carbonosas evidencian la acción del fuego. En Cerro Pintado (Cholila), debido a la particular posición topográfica del sitio la acción del agua no se manifestó tan intensamente como en el valle del río Manso, pero sí se advierten algunos de los daños señalados como producidos por el fuego. Una característica común a todos los sitios de la región es el alto grado de fragmentación de las muestras (por ejemplo, el 69,2\% de los tiestos de Paredón Lanfré tiene menos de $3 \mathrm{~cm}^{2}$-Bellelli, 2020a). Estas características, compartidas con conjuntos cerámicos de ambiente de bosque de Neuquén y Río Negro, contrastan con aquellos provenientes de sitios de estepa donde se espera encontrar en estratigrafía tiestos bien preservados, sin mayores alteraciones superficiales (Fernández \& Vitores, 2008).

Las características tecnológicas principales de la cerámica de este sector del bosque cordillerano fueron obtenidas por medio del análisis macroscópico de toda la muestra de los sitios del valle del río Manso en los que se recuperó este tipo de evidencia (Paredón Lanfré, Población Anticura, Casa de Piedra Carro y Campamento Argentino y un hallazgo aislado de una vasija casi completa). Fue complementado con el análisis petrográfico de una muestra seleccionada de Paredón Lanfré, Casa de Piedra Carro y del hallazgo aislado (Solá, 2010; Bellelli, 2020a, 2020b). Estos análisis mostraron una importante variabilidad tecnológica que se resume en once agrupamientos, algunos de los cuales están representados por pocos tiestos, generalmente de una misma pieza (Bellelli, 2020b). Así, el análisis de la matriz y de la composición de los antiplásticos y su representación porcentual en cada agrupamiento, mostró que la mayoría de ellos tienen un origen volcánico, con el agregado de arenas que podrían ser locales. En menor medida se registraron pastas donde el origen de minerales y rocas es granítico. En ambos tipos de pasta suelen, en algunos casos, observarse fragmentos de tiestos. Estas características, junto con la presencia de vidrio en la arcilla estaría mostrando estrechas semejanzas en la pasta con piezas estudiadas en el área de Traful (Aldazábal, Silveira \& Micaeli, 2008-2009; Solá, 2010; Bellelli 2020a, 2020b).

La comparación macroscópica de estas piezas del valle del río Manso con las de los sitios del valle del río Epuyén y de Cholila que completan el registro cerámico de la región, mostró que comparten las características tecnológicas de composición de la pasta y tratamiento de superficies. En la muestra de los tres sectores mencionados predominan los fragmentos de cuerpo, hay pocos bordes y asas y un muy escaso número de bases, todos, como se dijo, de tamaños pequeños. También se han recuperado fragmentos de torteros y de piezas cilíndricas pequeñas de función no definida. La decoración por incisión e impresión está presente en Cholila y en el río Manso. En esta última área la técnica más frecuente es el acanalado y también hay escasos tiestos con pintura que en algunos casos es resistente, lo cual vuelve a acercar estos conjuntos a los recuperados más al norte. Estas últimas técnicas están ausentes en Cholila y en el sitio del valle del Epuyén donde no se registraron piezas con ningún tipo de decoración (Figura 2) (Bellelli et al., 2003, 2007; Podestá et al., 2007; Bellelli, 2020a, 2020b).

\section{La Muestra}

La muestra que se envió a datar corresponde a residuos orgánicos adheridos a cinco tiestos que son parte de un conjunto más numeroso que fuera analizado desde una perspectiva isotópica en un estudio previo (Chaile et al. 2018). En el caso de Paredón Lanfré, el tiesto datado proviene del sector principal de la excavación, un depósito con baja integridad y escasa resolución cronológica, en el que los materiales arqueológicos se dan sin solución de continuidad a lo largo de 1,5 metros de sedimento. El inicio de la ocupación está datado alrededor de 1500 años ${ }^{14} \mathrm{C}$ AP, a partir de tres fechados efectuados sobre carbones recuperados en dos estructuras de combustión ubicadas en la base de la ex- 

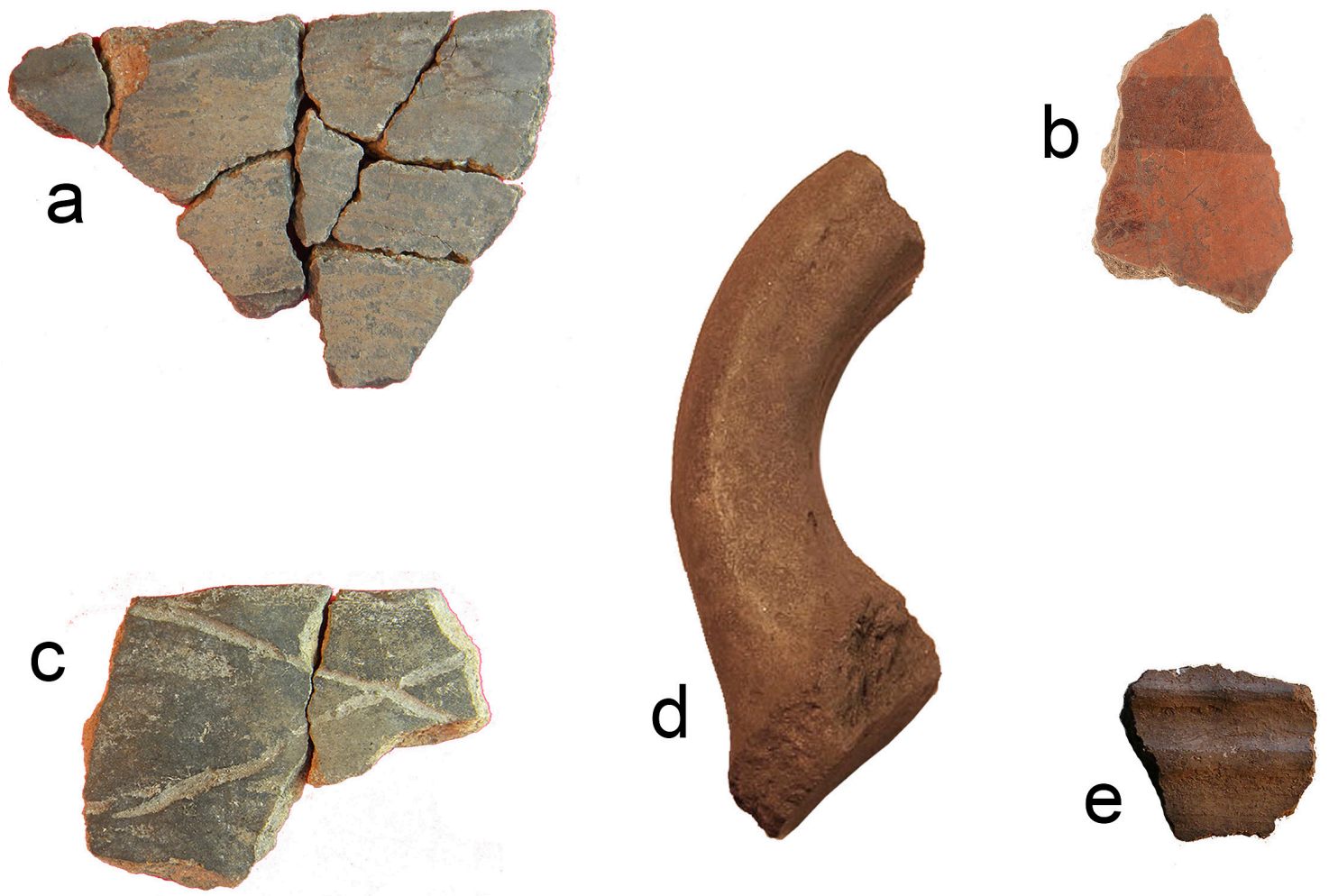

Figura 2. Se ilustran algunos ejemplos de la evidencia cerámica recuperada en Paredón Lanfré. A) siete fragmentos de cuerpo remontados. B) fragmento con pintura resistente. C) dos fragmentos de cuerpo con decoración incisa. D) asa. E) tiesto con decoración "acanalada".

cavación. Otros cuatro fechados datan acumulaciones de carbón en $1030 \pm 70,930 \pm 60,790 \pm 60$ y $490 \pm 60$ años ${ }^{14} \mathrm{C} \mathrm{AP}^{1}$ (Bellelli et al., 2007; Fernández, Bellelli, Carballido Calatayud, Podestá \& Vasini, 2010; Fernández et al., 2013). Estas dataciones están distribuidas a lo largo de la secuencia estratigráfica y tienen escaso valor para establecer asociaciones cronológicas con los materiales adyacentes. Algunas presentan inversiones en la posición estratigráfica y todas se asocian a materiales modernos, como trozos de plástico, metales y huesos de oveja (Andrade \& Fernández, 2017).

La cerámica del sitio Campamento Argentino proviene de un depósito de $70 \mathrm{~cm}$ de espesor fechado en $230 \pm 70$ y 560 \pm 60 años ${ }^{14} \mathrm{C} \mathrm{AP}$, dataciones obtenidas sobre acumulaciones de carbón vegetal (Fernández et al., 2013). Durante la excavación no se identificaron estructuras de combustión claras ni se recuperaron materiales con modificaciones antrópicas pasibles de ser fechados. Por otro lado, el hecho de que el carbón datado no haya sido recuperado en fogones debilita la relación entre materiales y cronología.

En el caso de Cerro Pintado los fechados provienen de estructuras de combustión y datan las ocupaciones entre
$1870 \pm 80$ y $680 \pm 60$ años ${ }^{14} \mathrm{C}$ AP. No obstante, el depósito arqueológico es considerado un palimpsesto, debido a la baja tasa de depositación de sedimentos (35 $\mathrm{cm}$ de potencia), la falta de indicios que permitan diferenciar niveles estratigráficos, las evidencias de pisoteo y la incidencia del uso actual del sitio sobre los materiales arqueológicos (Bellelli et al., 2003).

\section{Los fechados obtenidos y sus aportes a escala local y regional}

Las dataciones se procesaron en el AMS Laboratory de la Universidad de Arizona, a partir de residuos orgánicos presentes en cinco tiestos (Tabla 1). Uno proviene de los primeros $10 \mathrm{~cm}$ de la estratigrafía del sitio Paredón Lanfré y no tiene fechado asociado. Otro tiesto proviene del nivel 125-135 del sitio Campamento Argentino, que fue fechado en $230 \pm 70{ }^{14} \mathrm{C}$ AP. Los tres restantes vienen de la capa 3 de Cerro Pintado, que posee un fechado informado como Moderno (Bellelli et al., 2003; Bellelli et al., 2007; Fernández et al., 2019b).

Estos resultados están en consonancia con los planteos pre- 
vios respecto de la complejidad de los depósitos arqueológicos en el bosque. En Paredón Lanfré, la fecha de $960 \pm 22$ años ${ }^{14} \mathrm{C}$ AP (1028 a $1157 \mathrm{Cal} \mathrm{dC})$ constituye la fecha más temprana para el uso de esta tecnología en nuestra área de investigación. Por otra parte, refuerza la idea de que el sitio constituye un palimpsesto y que en él operaron procesos que favorecieron el desplazamiento vertical de los materiales. Este tiesto no sólo se ubica cerca de la superficie sino que está por encima del resto de los fechados (Figura 3). No obstante, la coincidencia cronológica entre la antigüedad de este tiesto y la acumulación de carbones datada en $930 \pm 60$ años ${ }^{14} \mathrm{C}$ AP indica de manera no ambigua el uso humano del sitio en ese momento. Así, quedarían definidos al menos tres momentos de uso de Paredón Lanfré: el más temprano alrededor de 1500 años AP, otro cercano a los 900 años y un tercero, en momentos históricos, evidenciado por el fechado de $330 \pm 50$ años ${ }^{14} \mathrm{C}$ AP y por el arte rupestre (Fernández et al., 2013, 2019b).

El tiesto de Campamento Argentino muestra que en este sitio también hay problemas de resolución cronológica. La fecha obtenida es de $578 \pm 20$ años ${ }^{14} \mathrm{C}$ AP (1315 a 1412 Cal dC.), casi 350 años más antigua que la datación disponible para el mismo nivel estratigráfico (Figura 4). Como en el caso anterior, se constata el desplazamiento vertical de materiales desde niveles más profundos hacia los más superficiales. Asimismo, la antigüedad de los residuos orgánicos del tiesto coincide con el fechado de $560 \pm 60$ años ${ }^{14} \mathrm{C}$ AP obtenido de una acumulación de carbones ubicada $50 \mathrm{~cm}$ más abajo. Esto permite definir el uso del sitio al menos en este momento.

A diferencia de los sitios de El Manso inferior, en Cerro Pintado la cronología se basa exclusivamente en fechados provenientes de fogones. Como ya señalamos, el problema aquí es la imposibilidad de relacionar materiales arqueológicos -incluida la cerámica- con cada uno de los fechados registrados. Las tres nuevas dataciones, cuyos rangos se superponen entre sí, aportan información novedosa sobre el uso de la tecnología cerámica en Cerro Pintado. Al mismo tiempo informan sobre la ocupación humana durante un lapso que hasta el momento no estaba identificado en el sitio (Figura 5). De acuerdo con el esquema cronológico que hemos planteado, se corresponde con lo que denominamos Tiempos históricos (Fernández et al., 2013, 2019b). El hallazgo de una primera falange de caballo ${ }^{2}$ en el mismo microsector y nivel que el tiesto datado en $328 \pm 31$ años ${ }^{14} \mathrm{C} \mathrm{AP}(1480$ a $1640 \mathrm{Cal}$
dC.) cobra otro significado a la luz de los nuevos datos y potencia la idea de la ocupación de Cerro Pintado durante este lapso. De esta forma, el sector sur de nuestra área de investigación se integra a lo observado en el valle del Manso inferior (Fernández et al., 2019b), en el que la ocupación humana se manifiesta como un proceso continuo a lo largo de los últimos 1700 años que incluye los momentos más recientes.

La cronología de uso de la cerámica obtenida en nuestro estudio coincide con la de otras muestras analizadas por el mismo método. Se trata de tiestos recuperados en contextos arqueológicos de la estepa de la Patagonia argentina. El más cercano, en términos geográficos, corresponde al sitio La Marcelina 1 (cuenca del Limay superior, Río Negro), con una cronología de 1040 \pm 70 años ${ }^{14} \mathrm{C}$ AP (Crivelli Montero et al., 2017). Hacia el sur, en la cuenca superior del Chalía (Chubut) las fechas registradas son de $471 \pm 38,461 \pm 35$ y $374 \pm 38$ años ${ }^{14} \mathrm{C}$ AP (Castro Esnal, Sacchi \& Pérez de Micou, 2011). Recientemente se dieron a conocer fechados de tiestos recuperados en superficie en el área comprendida entre el extremo sur del Macizo del Deseado y el río Santa Cruz de $420 \pm 20{ }^{14} \mathrm{C}$ años AP y $140 \pm 31{ }^{14} \mathrm{C}$ años AP (Cirigliano, Montenegro, Franco, Brook \& Cherkinsky, 2019). En el centro-oeste de Santa Cruz, las dataciones disponibles son de $886 \pm 82,373 \pm 45$ y $109 \pm 37$ años ${ }^{14} \mathrm{C}$ AP (Cassiodoro \& Tessone, 2014). Al igual que lo observado en El Manso inferior y Cholila son pocas las fechas más tempranas y predominan las posteriores a 500 años AP.

Para las zonas de bosque ubicadas inmediatamente al norte y al sur de nuestra área de investigación, la comparación sólo puede efectuarse utilizando los fechados del contexto de hallazgo ya que no se han datado residuos orgánicos. En general la cronología coincide con la antigüedad que hemos informado en este trabajo. Así, hacia el norte, en sitios de bosque del Lago Traful, la cronología más antigua registrada hasta el momento es de $1510 \pm 90$ años ${ }^{14} \mathrm{C}$ AP (Alero Los Cipreses, Silveira, 1996). A partir de este momento, tanto en el área del lago Traful como en la localidad arqueológica Meliquina, se incrementa el número de sitios con evidencia cerámica (Pérez, 2010, 2011; Silveira, López \& Aldazábal, 2013, entre otros), mientras que para el vecino Parque Nacional Nahuel Huapi se registra un fechado más tardío $\left(640 \pm 60\right.$ años ${ }^{14} \mathrm{C}$ AP) (Hajduk et al., 2006). Así, hacia el sur, en el Parque Nacional Los Alerces, Arrigoni (2002) obtuvo datacio-

\begin{tabular}{|c|c|c|c|c|c|c|c|c|}
\hline Localidad & Sitio & \#Pieza & Capa & $\begin{array}{l}\text { Cuadrícula / } \\
\text { Microsector }\end{array}$ & Fechado & $\delta^{13} \mathrm{C}$ & $\begin{array}{c}\text { Calibración } 2 \text { sigmas Años d.C. } \\
(95.4 \%)\end{array}$ & $\begin{array}{l}\text { Código } \\
\text { Laboratorio }\end{array}$ \\
\hline \multirow{2}{*}{ El Manso } & PL & $266-3$ & $0-10$ & G24A & $960 \pm 22$ & $-24,6$ & $\begin{array}{l}1028 \text { AD a } 1054 \text { AD }(20.4 \%) \\
1063 \text { AD a } 1157 \text { AD }(75.0 \%)\end{array}$ & AA109952 \\
\hline & CA & $15 / 2$ & 2 & E12D & $578 \pm 20$ & $-23,1$ & $\begin{array}{l}1315 \mathrm{AD} \text { a } 1362 \mathrm{AD}(66.1 \%) \\
1386 \mathrm{AD} \text { a } 1412 \mathrm{AD}(29.3 \%)\end{array}$ & AA109955 \\
\hline \multirow{3}{*}{ Cholila } & $\mathrm{CP}$ & 61 & 3 & G17C & $369 \pm 21$ & $-27,3$ & $\begin{array}{c}1455 \mathrm{AD} \text { a } 1524 \mathrm{AD}(56.1 \%) \\
1560 \mathrm{AD} \text { a } 1564 \mathrm{AD}(0.8 \%) \\
1570 \mathrm{AD} \text { a } 1631 \mathrm{AD}(38.5 \%)\end{array}$ & AA109953 \\
\hline & $\mathrm{CP}$ & 72 & 3 & G17A & $374 \pm 20$ & $-25,2$ & $\begin{array}{l}1454 \mathrm{AD} \text { a } 1522 \mathrm{AD}(61.9 \%) \\
1575 \mathrm{AD} \text { a } 1625 \mathrm{AD}(33.5 \%)\end{array}$ & AA109954 \\
\hline & $\mathrm{CP}$ & 184 & 3 & G16B & $328 \pm 31$ & $-27,2$ & $1480 \mathrm{AD}$ a $1640 \mathrm{AD}(95.4 \%)$ & AA109951 \\
\hline
\end{tabular}

Tabla 1. Fechados obtenidos por medio de la datación de residuos orgánicos depositados en tiestos de sitios ubicados en el bosque cordillerano norpatagónico. La calibración se realizó por medio del programa Oxcal 4.3.2 (Bronk Ramsey, 2020). Referencias: PL: Paredón Lanfré. CA: Campamento Argentino. CP: Cerro Pintado. 

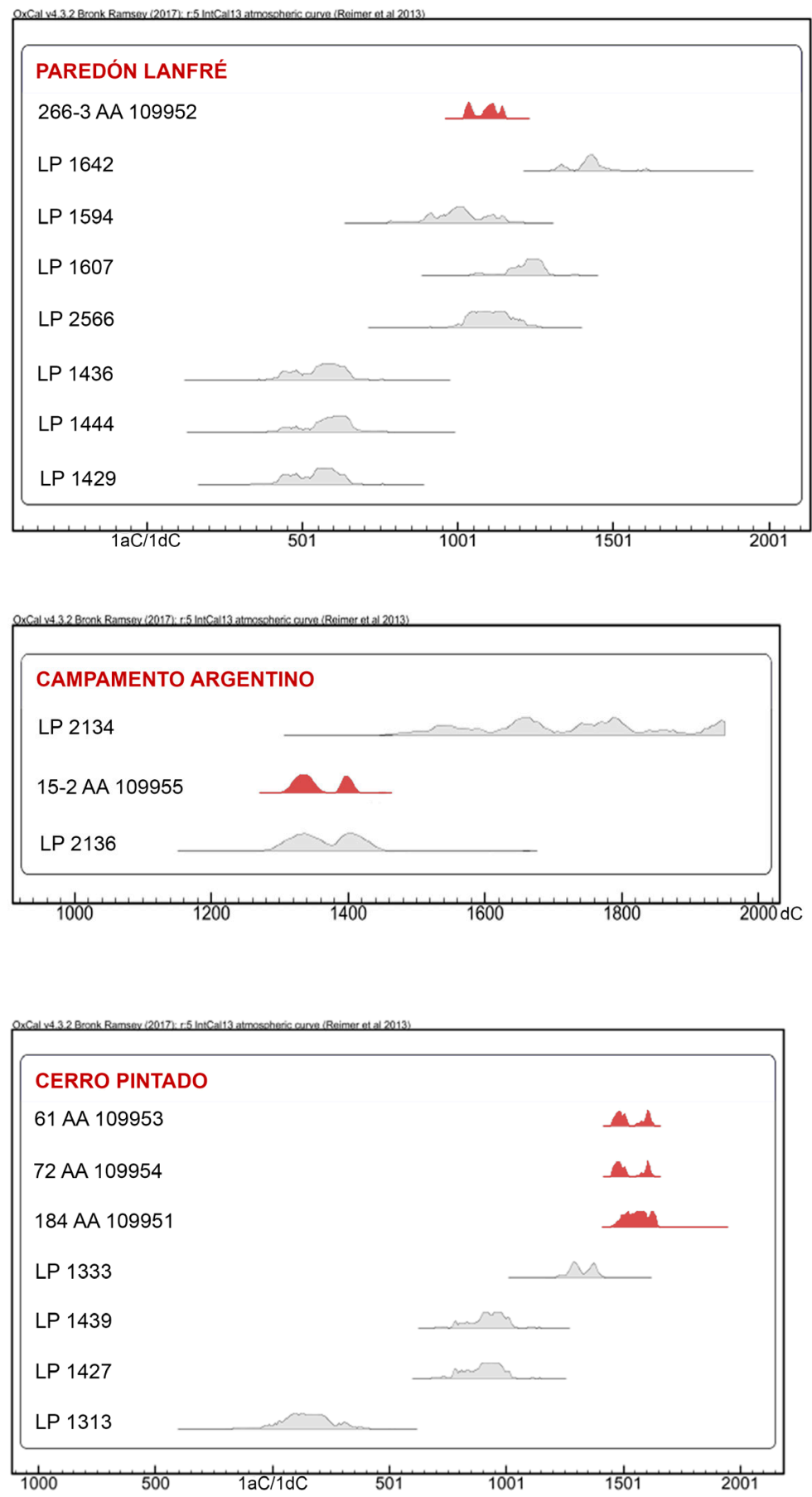

Figura 3. Fechados calibrados del sector principal de excavación del sitio Paredón Lanfré, ordenados según su posición en la estratigrafía (Fernández et al. 2013). En la versión on line se destaca en rojo la fecha que se presenta en este trabajo mientras que en la versión impresa está en un tono más oscuro.
Figura 4. Fechados calibrados del sitio Campamento Argentino, ordenados según su posición en la estratigrafía (Fernández et al. 2013). En la versión on line se destaca en rojo la fecha que se presenta en este trabajo mientras que en la versión impresa está en un tono más oscuro.
Figura 5. Fechados calibrados del sitio Cerro Pintado, ordenados según su posición en la estratigrafía (Bellelli et al. 2003). En la versión on line se destacan en rojo las fechas que se presentan en este trabajo mientras que en la versión impresa están en un tono más oscuro. nes de $740 \pm 70$ y $400 \pm 40$ años ${ }^{14} \mathrm{C} \mathrm{AP}$ en contextos cerámicos del sitio Alero Sendero de Interpretación.

\section{Palabras finales}

Finalmente, es necesario relacionar los resultados de nuestro trabajo con el estudio que realizaron Chaile y colaboradores sobre la composición isotópica de los residuos orgánicos datados. Estos autores indican que, salvo una excepción, los contenedores cerámicos del bosque se usaron para procesar principalmente proteínas y grasas de huemul (Hippocamelus bisulcus), y en menor proporción, roedores caviomorfos y plantas (Chaile et al., 2018). Gracias a los nuevos fechados estas acciones humanas se sitúan en el lapso en que el bosque fue ocupado a través de visitas más asiduas y/o más prolongadas. Cabe señalar que si el bosque hubiera sido explotado de forma logística desde la estepa, la señal isotópica debería ser más heterogénea, ya que podría reflejar tanto el consumo de recursos locales como el transporte desde la estepa de nutrientes -carnes y grasas- y/o de los ceramios. 
Así, la combinación de la información isotópica y cronológica continúa validando el último tramo del modelo de poblamiento propuesto para el área (Fernández et al., 2013). Además, las precisiones cronológicas alcanzadas a partir de estos cinco fechados contribuyen a comprender mejor la complejidad de los procesos de formación del registro en los sitios de bosque, alertan sobre la necesidad de valorar críticamente los materiales empleados para datar los contextos de este ambiente, y obligan a ponderar cuidadosamente las asociaciones entre los fechados y los materiales contenidos en los depósitos arqueológicos.

\section{Agradecimientos}

A Patricia Solá, quien aportó su gran experiencia y conocimientos para definir la estructura tecnológica de la muestra y su generosidad a la hora de compartir conocimientos e información. A las editoras de este volumen por la invitación y por su enorme paciencia. A los evaluadores por sus cuidadosos comentarios y valiosas sugerencias que ayudaron a mejorar el trabajo. A Ana Forlano por su gran ayuda en la realización de las figuras. Y a los pobladores y amigos del valle del río Manso y de Cholila que nos han brindado un enorme apoyo de todo tipo a lo largo de numerosas campañas. Los trabajos fueron realizados en el marco del PIP Conicet 365/2014 “Arqueología y antropología en el Suroeste de Río Negro y Noroeste de Chubut, Patagonia argentina. Segunda etapa.

\section{Notas}

1 La fecha más reciente obtenida en este sitio es de $330 \pm 50$ años ${ }^{14} \mathrm{C}$ AP y proviene de carbones dispersos vinculados a materiales arqueológicos recuperados en otro sector excavado, la cuadrícula F19 (Fernández et al. 2010).

2 Este hallazgo había sido informado previamente (Fernández 2010) pero no había sido interpretado dado que es el único hueso de esta especie registrado en el sitio y que no podía descartarse su carácter intrusivo.

\section{Bibliografía}

Andrade, A. \& Fernández, P. M. (2017). Rodent consumption by hunter-gatherers in north Patagonian Andean forests (Argentina): Insights from the small vertebrate taphonomic analysis of two late Holocene archaeological sites. Journal of Archaeological Science: Reports 11, 390-399.

Arrigoni, G. (2002). Los ceramistas prehistóricos del valle del río Desaguadero, Parque Nacional Los Alerces. Provincia del Chubut. Relaciones 27, 395-412.

Aldazábal, V., Silveira, M. \& Micaeli, A. (2008-2009). La cerámica del sitio Alero Las Mellizas, Lago Traful, Neuquén. Anales de Arqueología y Etnología, 63-64, 177-195.

Bellelli, C. (2020a). La cerámica acanalada de Norpatagonia. Caracterización y cronología en el valle del río Manso (Río Negro). En V. Schuster \& A. E. Pérez (Eds.), Cerámica arqueológica Patagonia, (pp. 95-114). Buenos Aires: Vazquez Mazzini Editores, Universidad Maimónides, Fundación Azara.

Bellelli, C. (2020b). Tiestos en el bosque. Hacia una caracterización de los conjuntos cerámicos del valle inferior del río
Manso (Provincia de Río Negro). Manuscrito inédito.

Bellelli, C., Carballido, M., Fernández, P. \& Scheinsohn, V. (2003). El pasado entre las hojas. Nueva información arqueológica del Noroeste de la provincia del Chubut, Argentina. Werken, 4, 25-42.

Bellelli, C., Carballido Calatayud, M., Fernández; P. \& Scheinsohn, V. (2007). Investigaciones arqueológicas en el valle del río Manso inferior (provincia de Río Negro). En Resúmenes ampliados, Actas del XVI Congreso Nacional de Arqueología Argentina, (pp. 309-314). Jujuy: Universidad Nacional de Jujuy, Facultad de Humanidades y Ciencias Sociales.

Bronk Ramsey, C. (2020). OxCal. 4.3.2. https://c14.arch.ox.ac. uk/oxcal.html, (acceso, 30 de julio, 2020).

Carballido Calatayud, M. \& Bellelli, C. (2020). Historias de agua y fuego. Modificaciones del material lítico en sitios del bosque norpatagónico. Revista del Museo de Antropología 13 (1), 347-354.

Cassiodoro, G. E. \& Tessone, A. (2014). Análisis radiocarbónico y de isótopos estables en residuos cerámicos del centro-oeste de Santa Cruz (Patagonia). Relaciones 39, 293-299.

Castro Esnal A., Sacchi, M. \& Pérez de Micou, C. (2011). Aspectos generales de la tecnología lítica de sitios de la Colonia El Chalía, SO de la provincia de Chubut, Patagonia, Argentina. International Journal of South American Archaeology, 9, 2840.

Cirigliano, N., Montenegro, T., Franco, N. V., Brook, G. A. \& Cherkinsky, A. (2019). Hallazgos cerámicos y su cronología en el área comprendida entre el extremo sur del Macizo del Deseado y la cuenca del río Santa Cruz (Patagonia sur, Argentina). Magallania, 47 (2), 133-149.

Crivelli Montero, E.A, Ramos, M., Cordero, J. A., Fernández, F. J., Vitores, M. \& Homar, A. (2017). Arqueología del cañadón Fta Miche, Provincia de Río Negro, noroeste de la Patagonia. Informe preliminar. En M.T. de Haro, A. M. Rocchietti, M. A. Runcio, M. V. Fernández y O. Hernández de Lara (Eds.), Anti. Latinoamérica: una mirada desde el presente hacia el pasado, (pp. 67-97). Buenos Aires: Aspha, Centro de Investigaciones Precolombinas.

Chaile, C., Tessone, A., Cassiodoro, G., Bellelli, C. \& Belardi, J. B. (2018). $\delta^{13} \mathrm{C}$ and $\delta^{15} \mathrm{~N}$ in organic residues of Patagonia pottery. Implications for studies of diet and subsistence strategies among late Holocene hunter-gatherers. Journal of Archaeological Science: Reports 20, 47-56

Fernández, M. \& Vitores, M. (2008). Distribución de la cerámica arqueológica en la cuenca superior y media del río Limay. En P. F. Azar, E. M. Cúneo \& S. Rodríguez (Eds.) Tras la senda de los ancestros: Arqueología de Patagonia. Actas de la mesa de arqueología de las $3^{\circ}$ Jornadas de Historia de la Patagonia. San Carlos de Bariloche, Río Negro, 6 - 8 de noviembre de 2008, CDRom. Neuquén: Universidad Nacional del Comahue,

Fernández, P. M. (2010). Cazadores y presas. 3500 años de interacción entre seres humanos y animales en el noroeste de Chubut. Buenos Aires: Fundación de Historia Natural Félix de Azara. 
Fernández, P. M. \& Tessone, A. (2014). Modos de ocupación del bosque patagónico de la vertiente oriental de los Andes: aportes desde la ecología isotópica. Revista Chilena de Antropología, 30, 83-89.

Fernández, P. M., Bellelli, C., Altinier, V., Pereyra, F., Scheinsohn, V., Carballido Calatayud, M. \& Podestá, M. (2008). Fuego, agua, tierra. Procesos de formación del registro arqueológico en el bosque caducifolio del noroeste de Patagonia. En Libro de resúmenes de las VII Jornadas de Arqueología de la Patagonia, (p. 49). Ushuaia: CADIC-CONICET.

Fernández, P. M., Bellelli, C., Carballido Calatayud, M., Podestá, M. \& Vasini, A. (2010). Primeros resultados de las investigaciones arqueológicas en el sitio Población Anticura (Río Negro, Argentina). En J. R. Barcena \& H. Chiavazza (Eds.), Arqueología Argentina en el Bicentenario de la Revolución de Mayo. XVII Congreso Nacional de Arqueología Argentina, (pp. 1895-1900). Mendoza: Facultad de Filosofía y Letras, Universidad Nacional de Cuyo.

Fernández, P. M., Carballido Calatayud, M., Bellelli, C. \& Podestá, M. M. (2013). Tiempo de cazadores. Cronología de las ocupaciones humanas en el valle del río Manso inferior (Río Negro). En A. F. Zangrando, R. Barberena, A. Gil, G. Neme, M. Giardina, L. Luna, C. Otaola, S. Paulides, L. Salgán \& A. Tivoli (Eds.), Tendencias teórico-metodológicas y casos de estudio en la arqueología de Patagonia, (pp.167-175). San Rafael: Museo de Historia Natural de San Rafael, SAA, INAPL.

Fernández, P. M., Carballido Calatayud, M., Bellelli, C., Tchilinguirián, P., Leonardt, S. \& Fernández M. (2019a). Nuevos datos sobre el poblamiento inicial del bosque del centro-norte de Patagonia. Latin American Antiquity, 30 (2), 300-317.

Fernández, P. M., Carballido Calatayud, M., Bellelli, C. \& Fernández, M. (2019b). Ocupaciones de tiempos históricos en el bosque del centro-norte de Patagonia Argentina. En J. Gómez Otero, A. Svoboda \& A. Banegas (Eds.), Arqueología de la Patagonia: el pasado en las arenas, (pp. 105-116). Puerto Madryn: Instituto de Diversidad y Evolución Austral, CONICET.

Giaché, Y. \& Bianchi, M. M. (2018). 13,000 years of forest history in the Río Manso Inferior Valley, Northern Patagonia. Fire-vegetation-humans links. Journal of Archaeological Science: Reports, 18, 769-774.

Hajduk, A., Albornoz, A. M. \& Lezcano, M. (2006). Levels with Extinct Fauna in the Forest Rockshelter El Trébol (Northwest Patagonia, Argentina). Current Research in the Pleistocene, $23,55-57$.

Iglesias, V., Whitlock, C., Bianchi, M. M., Villarosa, G. \& Outes, V. (2012). Holocene climate variability and environmental history at the Patagonian forest/steppe ecotone: Lago Mosquito $\left(42.50^{\circ} \mathrm{S}, 71.40^{\circ} \mathrm{W}\right)$ and Laguna del Cóndor $\left(42.20^{\circ} \mathrm{S}\right.$, $\left.71.17^{\circ} \mathrm{W}\right)$. The Holocene, 22, 1297-1307.

Pérez, A. (2010). Arqueología del bosque. El registro arqueológico del interior y borde de bosque de Norpatagonia. En Actas XVII Congreso Nacional de Arqueología Chilena, (pp.15151528). Sociedad Chilena de Arqueología.

Pérez, A. (2011). Algunas reflexiones sobre la alfarería del cen- tro sur de Chile y ambientes lacustres precordilleranos de la Patagonia septentrional argentina. En P. Navarro Floria \& W. Del Río (Eds.), Cultura y espacio. Araucanía-Norpatagonia, (pp. 293-314). Bariloche: Instituto de Investigaciones en Diversidad Cultural y Procesos de Cambio. Universidad Nacional de Río Negro.

Podestá, M., Bellelli, C., Fernández, P., Scheinsohn, V., Carballido Calatayud, M., Forlano, A., Marchione, P., Tropea, E., Vasini, A., Alberti, J., Gallo, M. \& Moscovici, G. (2007). Arqueología del valle del río Epuyén (El Hoyo, Chubut, Patagonia argentina). En F. Morello, M. Martinic, A, Prieto \& F. Bahamonde (Eds.), Arqueología de Fuego-Patagonia. Levantando piedras, desenterrando huesos...y develando arcanos, (pp. 427-442). Punta Arenas: Ediciones CEQUA. Centro de Estudios del Hombre Austral, Instituto de la Patagonia-Universidad de Magallanes.

Silveira, M. (1996). Alero Los Cipreses (pcia. de Neuquén). En J. Gómez Otero (Ed.) Arqueología, sólo Patagonia, (pp.107118). Ponencias de las 2 das. Jornadas de Arqueología de la Patagonia, 1993, Puerto Madryn.

Silveira M., López, L. \& Aldazábal, V. (2013). El uso del espacio durante el Holoceno tardío -últimos 3500 años- en el bosque andino de Patagonia septentrional, Lago Traful, sudoeste de la provincia de Neuquén. Anuario de Arqueología, 5, 85-101.

Solá, P. (2010). Análisis microscópico de cerámica arqueológica. Proyecto de Investigación Arqueológica en la región del Valle del Río Manso (Provincia de Río Negro - Argentina). Manuscrito inédito.

Whitlock, C., Bianchi, M.M., Bartlein, P.J., Markgraf, V., Marlon, J., Walsh, M. \& Mccoy, N. (2006). Postglacial vegetation, climate, and fire history along the east side of the Andes (lat 41-42.5 S), Argentina. Quaternary Research, 66, 187.201. 